\title{
Scavenging of lipid peroxyl radicals protects plasma lipids and proteins from peroxynitrite
}

\author{
AYMAN G. MUSTAFA ${ }^{1,3}$, MAHMOUD A. ALFAQIH ${ }^{2}$, OTHMAN AL-SHBOUL ${ }^{2}$ and AHMED AL-DWAIRI ${ }^{2}$ \\ Departments of ${ }^{1}$ Anatomy and ${ }^{2}$ Physiology and Biochemistry, School of Medicine, \\ Jordan University of Science and Technology, Irbid 22110, Jordan
}

Received June 6, 2018; Accepted September 5, 2018

DOI: $10.3892 /$ br.2018.1144

\begin{abstract}
Peroxynitrite can be produced in the vasculature from a superoxide anion reaction with nitric oxide. A surplus of peroxynitrite in the intravascular compartment is a common feature of several chronic diseases. The development of pharmacological modalities that interfere with the formation of peroxynitrite or inhibit its oxidative damage may be of utility for the prevention and/or treatment of several pathologies. Our previous investigations showed that catalytically inactivating peroxynitrite-derived free radicals with tempol or scavenging reactive aldehyde species with phenelzine protects the blood plasma and platelets from the oxidative damage of peroxynitrite. However, the degree of inhibition of the cytotoxic effects of peroxynitrite using tempol or phenelzine was modest. In the present study, the aim was to examine if scavenging lipid peroxyl radicals with U-83836E can achieve superior protection from peroxynitrite. This was assessed by treating blood plasma or platelets with $100 \mu \mathrm{M}$ peroxynitrite alone or in combination with U-83836E, and then measuring the levels of thiobarbituric acid reactive substances (TBARS) and protein carbonyl formation as indices of lipid peroxidation and protein oxidation, respectively. It was observed that scavenging lipid peroxyl radicals with $75-100 \mu \mathrm{M}$ U-83836E increasingly reversed protein carbonylation induced by peroxynitrite in
\end{abstract}

Correspondence to: Dr Mahmoud A. Alfaqih, Department of Physiology and Biochemistry, School of Medicine, Jordan University of Science and Technology, P.O. Box 3030 Ar Ramtha, Irbid 22110, Jordan

E-mail: maalfaqih@just.edu.jo

Present address: ${ }^{3}$ Department of Basic Medical Sciences, College of Medicine, Qatar University, Doha, Qatar

Abbreviations: ROS, reactive oxygen species; PUFA, polyunsaturated fatty acids; DNA, deoxyribonucleic acid; TBARS, thiobarbituric acid reactive substances; IRB, Institutional Review Board; CPDA, citrate phosphate dextrose adenine; SPSS, Statistical Package for Social Studies; ELISA, enzyme-linked immunosorbent assay; 4-HNE, 4-hydroxynonenal; MDA, malondialdehyde

Key words: peroxynitrite, oxidative stress, reactive oxygen species, lipid peroxyl radicals, thiobarbituric acid reactive substances blood plasma and platelets, in addition to TBARS formation in blood plasma. These findings are further discussed in the context of the mechanisms by which U-83836E may protect against the cell-damaging effects of peroxynitrite.

\section{Introduction}

Aerobic organisms produce reactive oxygen species (ROS) during normal physiological processes, including cellular respiration (1), and as a means to combat foreign microorganisms during inflammation (2). However, if produced in mass quantities, ROS may cause considerable tissue damage (3). Therefore, aerobic organisms have evolved several mechanisms that antagonize the potentially damaging effects of ROS (4), including glutathione (5), superoxide dismutase (6) and catalase (6) systems. The above defense mechanisms can become overwhelmed by the ROS surplus leading to a state commonly referred to as 'oxidative stress' (7). This state is considered to contribute to the development and maintenance of several chronic disease conditions, including cancer (8), Alzheimer's disease (9), traumatic brain injury $(10,11)$, and insulin resistance (12), and to ageing $(7,13)$.

In view of the central role of ROS in the etiology of several pathologies, the mechanism by which ROS produce cellular damage has been and remains the subject of extensive investigation. Although the exact mechanism remains to be elucidated, several lines of evidence from several research groups in the field indicate that ROS can trigger cellular damage through i) the peroxidation of polyunsaturated fatty acids of cellular membranes (14), ii) the generation of DNA mutations through the nitration and deamination of DNA (15), and iii) protein nitration and carbonylation (16), which eventually disrupts cellular function. Noteworthy, the majority of the ROS are involved in chain reactions that result in the formation of new ROS species and further propagation of the initial damage (17). In addition, several of the cell damaging effects of ROS species can be attributed to the decomposition of the parent ROS into other markedly reactive radicals (18). Given the above discussion, therapeutic interventions that interfere with ROS production and/or propagation may be of benefit for the treatment of several chronic diseases linked to 'oxidative stress'. In the intravascular compartment, pharmacological agents that reduce the cell damaging effects of peroxynitrite are a suitable target for therapy for several reasons; peroxynitrite 
is the most regularly produced reactive species in the intravascular compartment (19), and the formation of peroxynitrite in the intravascular compartment has been linked to the etiology of several pathologies (19).

In our two previous reports, an in vitro system was discussed that permitted the investigation of the damaging effects of peroxynitrite on the proteins and lipids of the intravascular compartment; specifically on blood plasma and isolated platelets. In the first report, it was shown that tempol, a drug that catalytically inactivates peroxynitrite-derived free radicals, antagonizes the effects of peroxynitrite on blood plasma and on platelet lipids and proteins (20). In the second report, it was shown that phenelzine, a scavenger of reactive aldehyde species, produced comparable effects to that of tempol (21). However, although the results in these two previous reports were encouraging, the degree of inhibition of the cell damaging effects via using tempol or phenelzine were modest and we were not able to completely reverse the oxidative damage induced by peroxynitrite on the examined endpoints; namely, thiobarbituric acid reactive substances (TBARS) and protein carbonylation. In the present study, it was hypothesized that, by using a different antioxidant that targets a different step of the proposed model by which peroxynitrite induces its cytotoxic effects, it is possible to achieve superior protection from peroxynitrite and completely reverse its cell damaging effects. Specifically, the present study examined the protective effects of U-83836E, a scavenger of lipid peroxyl radicals (22), against the peroxynitrite-mediated oxidative damage of blood proteins and lipids. The mechanism/s by which U-83836E protects against the cell damaging effects of peroxynitrite is further discussed.

\section{Materials and methods}

Description of study participants and sample collection. The present study was a prospective study performed in the family medicine clinics of King Abdullah University Hospital (KAUH; Ramtha, Jordan). KAUH is a tertiary teaching hospital affiliated with Jordan University of Science and Technology (Irbid, Jordan). Following approval of the study from the Institutional Review Board (IRB) of KAUH (IRB no. 1/105/2017), 1 unit of blood was collected from each of three eligible participants. The selection of study participants was based on the criteria of being of Jordanian descent and 22-25 years of age. Any individual who was a smoker or an ex-smoker, suffered from chronic illnesses associated with increased ROS, including hypertension, type 1 or type 2 diabetes mellitus, atherosclerosis or dyslipidemia, or received anti-histamines or nonsteroidal anti-inflammatory drugs prior to blood collection (up to 2 weeks) were not invited to participate in the study. A total of 2 males and 1 female consented to participate. Blood withdrawal was performed between October and December, 2014. Bags containing CPDA-1 anticoagulant were used for blood collection. In order to separate blood plasma and platelets, whole blood collected in the above bags was divided into six individual CPDA1 tubes. This number corresponds to the number of experimental groups.

Isolation of plasma and platelets from whole blood. The methods used to recover plasma or platelets were as previously described (20). In brief, whole blood was centrifuged at $450 \mathrm{x} \mathrm{g}$ for $6.20 \mathrm{~min}$ at room temperature to recover platelet-rich plasma. The platelet-rich plasma recovered from the previous step was then subjected to centrifugation at 2,500 $\mathrm{x} g$ for $5.40 \mathrm{~min}$ at room temperature, which caused the sedimentation of a platelet rich pellet. This pellet was then washed twice with Tyrode's buffer and then re-suspended in the same buffer. The final platelet number was measured with an automated cell counter (Thermo Fisher Scientific, Inc., Waltham, MA, USA) and adjusted to a final platelet count of $10 \% / \mathrm{ml}$.

Sample treatment. The experimental design and sample treatments were as previously described (20). In brief, the plasma or isolated platelets were distributed into six-well plates ( $2 \mathrm{ml} /$ well). Each well corresponded to one of the CPDA1 tubes described above. The samples in the wells were then treated with either vehicle $(2 \mu \mathrm{l} 0.3 \mathrm{M} \mathrm{NaOH}), 100 \mu \mathrm{M}$ peroxynitrite (cat. no. 81565; Cayman Chemical, Ann Arbor, MI, USA), or a combination of peroxynitrite with different doses of U-83836E (25, 50, 75 or $100 \mu \mathrm{M}$; Biomol International, LP, Plymouth Meeting, PA, USA). All treatments lasted for $20 \mathrm{~min}$ at $37^{\circ} \mathrm{C}$.

Estimation of total plasma proteins. The protein concentration of the plasma samples was measured using a Bradford protein assay (Bio-Rad Laboratories, Inc., Hercules, CA, USA) according to the manufacturer's protocol. The absorbance was determined at $595 \mathrm{~nm}$ on an ELx800 ELISA reader (BioTek Instruments, Inc., Winooski, VT, USA).

Detection of carbonyl group and TBARS. The methods used for the detection of carbonyl groups and TBARS were detailed previously (20) and are thus only briefly discussed in the present study. In terms of protein carbonylation, an enzyme-linked immunosorbent assay (ELISA)-based assay was used to determine the quantity of carbonyl groups in each sample (cat. no. STA-310; Cell Biolabs, Inc., San Diego, CA, USA). In this assay, the carbonyl groups of proteins were allowed to react with dinitrophenyl hydrazine (DNPH), which produces a dinitrophenyl hydrazone derivative. The excess DNPH was then washed with a PBS/ethanol solution. The quantity of the resulting hydrazone derivative was then quantified through its reaction with anti-DNP antibody (rabbit immunoglobulin G; cat. no. 231002). A total of $100 \mu$ l of the primary antibody was used at a final dilution of 1:1,000. The antibody was incubated for $1 \mathrm{~h}$ at room temperature on an orbital shaker. The absorbance was measured at $450 \mathrm{~nm}$ on an ELx800 microplate reader (BioTek Instruments, Inc.). The TBARS assay quantifies the levels of malondialdehyde (MDA) and other minor aldehyde species through their reaction with thiobarbituric acid (TBA). MDA is a reactive aldehyde species and a byproduct of lipid peroxidation. In the present study, a colorimetric based assay was used to measure the levels of TBA-MDA adduct of each sample (cat. no. ab118970; Abcam, Cambridge, UK). In this assay, the samples were first allowed to react with $\mathrm{H}_{2} \mathrm{SO}_{4}$ and phosphotungstic acid. This step causes precipitation of the lipids in the sample. Following a centrifugation step (13,000 x g for $3 \mathrm{~min}$ at room temperature), the lipid pellet was collected and then resuspended with water. TBA solution $(600 \mu \mathrm{l})$ was then added to the dissolved pellet and boiled for $1 \mathrm{~h}$. The reaction was then cooled down in an ice bath for $10 \mathrm{~min}$. The quantity of MDA-TBA adduct was finally 
measured spectrophotometrically at $530 \mathrm{~nm}$ on an ELx800 microplate reader (BioTek Instruments, Inc.).

Statistical analysis. Statistical analysis was performed using Statistical Package for Social Studies software version 17 (SPSS, Inc. Chicago, IL, USA). One way analysis of variance was used to compare between the different experimental groups, followed by Fisher's post-hoc test. $\mathrm{P}<0.05$ was considered to indicate a statistically significant difference between the groups.

\section{Results}

U83836E antagonizes the oxidative damage induced by peroxynitrite on the proteins of blood plasma and platelets. In our previous study, it was demonstrated that peroxynitrite induced oxidative damage of blood plasma and platelet proteins when protein oxidative damage was evaluated by measuring carbonyl group formation following peroxynitrite treatment (20). The present study aimed to determine whether U83836E has the ability to antagonize the oxidative damaging effects of peroxynitrite on blood plasma and platelets proteins. Therefore, blood plasma and platelet samples were treated with $100 \mu \mathrm{M}$ of peroxynitrite alone or in combination with the different doses of $\mathrm{U}-83836 \mathrm{E}(25,50,75$ or $100 \mu \mathrm{M})$, and protein carbonyl formation was then measured in the treated samples (Figs. 1 and 2). It was found that U-83836E significantly $(\mathrm{P}<0.05)$ and in a dose-dependent manner reduced the rate of carbonyl group formation of plasma proteins induced by peroxynitrite treatment at doses of 50,75 or $100 \mu \mathrm{M}$ (Fig. 1). In addition, it was found that U-83836E treatment significantly $(\mathrm{P}<0.05)$ reduced the effects of peroxynitrite treatment on platelet proteins at all the doses assessed (25, $50,75$ or $100 \mu \mathrm{M})$. Of note, in contrast to the plasma-treated samples, U-83836E was able to completely reverse the effect of peroxynitrite treatment at U-83836E doses of 75 or $100 \mu \mathrm{M}$.

U83836E antagonizes the oxidative damage induced by peroxynitrite on the lipids of blood plasma and platelets. An increase in TBARS concentration is a hallmark of lipid peroxidative damage. In our previous study, it was shown that peroxynitrite at a dose of $100 \mu \mathrm{M}$ significantly increased the TBARS concentration in blood plasma and platelets, indicating an increase in lipid peroxidation (20). The present study aimed to evaluate whether U-83836E can antagonize the oxidative effects of peroxynitrite on blood plasma and platelet lipids using TBARS concentration as an endpoint. Therefore, blood plasma and platelet samples were treated with $100 \mu \mathrm{M}$ of peroxynitrite alone or in combination with different doses of U-83836E $(25,50,75$ or $100 \mu \mathrm{M})$, and the TBARS concentrations in the treated samples were then then measured (Figs. 3 and 4). It was found that U-83836E significantly $(\mathrm{P}<0.05)$ and in a dose-dependent manner reduced the TBARS concentration in the blood plasma (Fig. 3) and platelet lipids (Fig. 4) induced by peroxynitrite treatment at U-83836E doses of 50, 75 or $100 \mu \mathrm{M}$. Of note, the antagonizing effect of $\mathrm{U}-83836 \mathrm{E}$, in terms of the percentage reduction in TBARS concentration, was more marked in the blood plasma-treated samples (Fig. 3) compared with that in the platelet samples (Fig. 4) at U-83836E doses of 50, 75 and $100 \mu \mathrm{M}$.

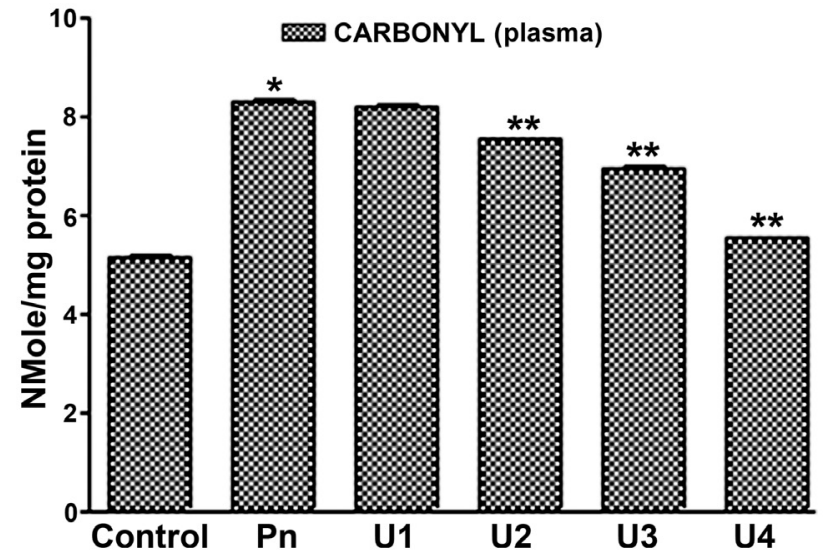

Figure 1. U83836E reduces the ability of Pn to induce protein carbonyl formation in plasma proteins. Plasma samples were treated with $100 \mu \mathrm{M}$ of Pn alone or in combination with U83836E at concentrations of $25,50,75$ or $100 \mu \mathrm{M}$, designated as U1, U2, U3 and U4 respectively. Protein carbonylation was later measured using a colorimetric based assay. The results are expressed as nmol of protein carbonyl group formation per $\mathrm{mg}$ of total protein. The results are representative of three independent experiments performed in triplicate. Protein carbonyl formation is expressed as the mean \pm standard deviation. ${ }^{*} \mathrm{P}<0.05$, compared with the control (no U83836E) group; ${ }^{* *} \mathrm{P}<0.05$, compared with the Pn group. Pn, peroxynitrite.

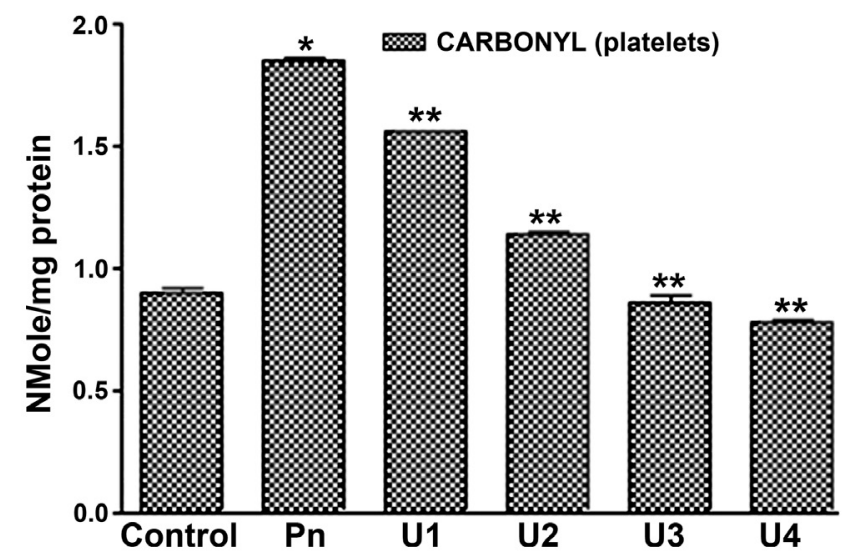

Figure 2. U83836E reduces the ability of Pn to induce protein carbonyl formation in platelet proteins. Platelet samples were treated with $100 \mu \mathrm{M}$ Pn alone or in combination with U83836E at concentrations of $25,50,75$ or $100 \mu \mathrm{M}$. designated as U1, U2, U3 and U4, respectively. Protein carbonylation was later measured using a colorimetric based assay. The results are expressed as $\mathrm{nmol}$ of protein carbonyl group formation per $\mathrm{mg}$ of total protein. The results are representative of three independent experiments performed in triplicate. Protein carbonyl formation is expressed as the mean \pm standard deviation. ${ }^{*} \mathrm{P}<0.05$, compared with the control (no U83836E) group; ${ }^{* *} \mathrm{P}<0.05$, compared with the Pn group. Pn, peroxynitrite.

\section{Discussion}

The production of peroxynitrite in the intravascular compartment is a hallmark and a consistent feature of a number of chronic diseases (19). Accordingly, attenuation of the oxidative damage induced by peroxynitrite in the vasculature remains one of the most important clinical challenges (23). However, despite the clear medical requirement for potent inhibitors of the oxidative effects of peroxynitrite on the blood, a review of the literature in the area of reactive oxygen and nitrogen species shows that the majority of the studies have been performed in the context of inhibiting the oxidative damage of 


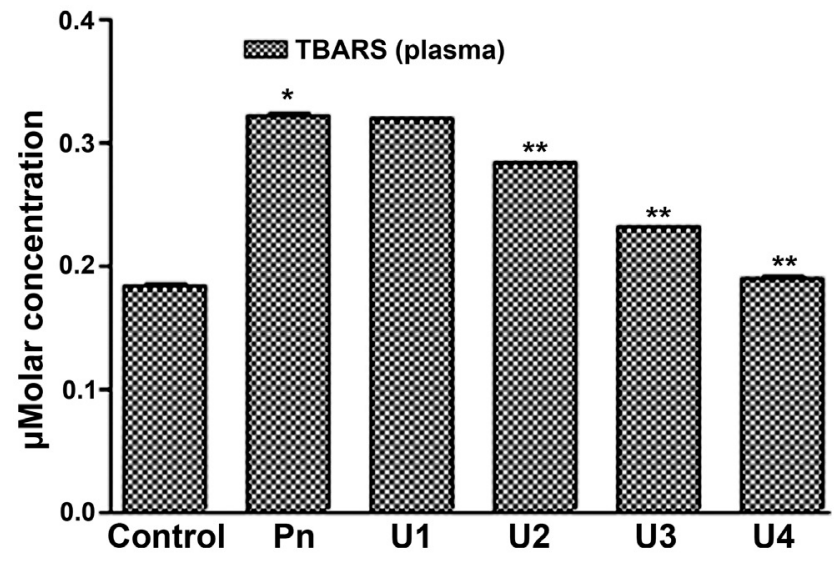

Figure 3. U83836E reduces the ability of peroxynitrite to induce TBARS formation in plasma lipids. Plasma samples were treated with $100 \mu \mathrm{M}$ of Pn alone or in combination with U83836E at concentrations of 25, 50, 75 or $100 \mu \mathrm{M}$, designated as $\mathrm{U} 1, \mathrm{U} 2, \mathrm{U} 3$ and $\mathrm{U} 4$, respectively. TBARS formation was then measured using a colorimetric based assay. The results are expressed as $\mu \mathrm{M}$ concentration of TBARS and are representative of three independent experiments performed in triplicate. The concentration of TBARS is expressed as the mean \pm standard deviation. " $\mathrm{P}<0.05$, compared with the control (no U83836E) group; ${ }^{* *} \mathrm{P}<0.05$, compared with the Pn group. TBARS, thiobarbituric acid reactive substances; Pn, peroxynitrite.

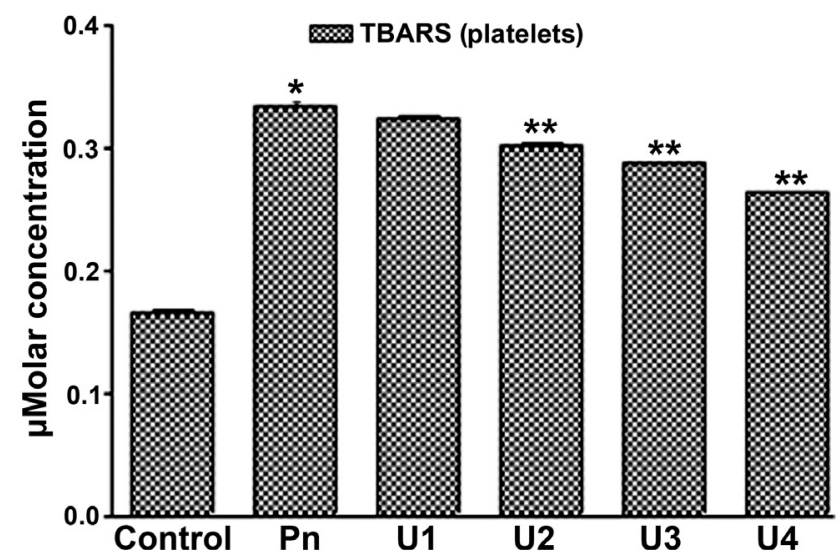

Figure 4. U83836E reduces the ability of Pn to induce TBARS formation in platelet lipids. Platelet samples were treated with $100 \mu \mathrm{M}$ of Pn alone or in combination with U83836E at concentrations of $25,50,75$ or $100 \mu \mathrm{M}$ designated as U1, U2, U3 and U4, respectively. TBARS formation was then measured using a colorimetric based assay. The results are expressed as $\mu \mathrm{M}$ concentration of TBARS and are representative of three independent experiments performed in triplicate. The concentration of TBARS is expressed as the mean \pm standard deviation. ${ }^{*} \mathrm{P}<0.05$, compared with the control (no U83836E) group; ${ }^{* *} \mathrm{P}<0.05$, compared with the Pn group. TBARS, thiobarbituric acid reactive substances; $\mathrm{Pn}$, peroxynitrite.

peroxynitrite in the nervous system (24). Our previous studies reported on the potential clinical utility of two important pharmacological agents as inhibitors of the cytotoxic effects of peroxynitrite on blood plasma and on platelet lipids and proteins; namely, tempol (20) and phenelzine (21). Although, these two agents significantly inhibited the oxidative damage induced by peroxynitrite on blood plasma and on platelet lipids and proteins. The inhibitory effects of the two agents were modest in certain endpoints investigated; for example, there remained a significant difference between the antioxidant treatment groups and the control group. Therefore, potential remains for identifying superior inhibitors. The present study hypothesized that, by investigating other antioxidant agents that interfere with other steps of the proposed model of how peroxynitrite induces its cytotoxic effect, it is possible to identify more potent inhibitors. Additionally, investigating other inhibitors is likely to provide further insight on the biochemical mechanisms underlying how peroxynitrite causes cell, body fluid and tissue damage. To this end, using an in vitro system previously developed for investigating the protective effects of tempol (20) and phenelzine (21), the present study investigated whether another antioxidant (U-83836E) was also able to inhibit the oxidizing effects of peroxynitrite on blood plasma and platelet lipids and proteins.

The structure of U-83836E, as reviewed previously (25), shows that it has the advantage of combining two different mechanisms for attenuating oxidative damage in one single structure. U-83836E is a 2-methylaminochroman, which combines the chroman ring of $\alpha$-tocopherol (vitamin E) with a bis-pyrrolo pyrimidine. The chroman ring component of U-83836E can effectively scavenge lipid peroxyl radicals. However, this results in the formation of the particularly weak chroman radical. Involvement in the subsequent 'scavenging cycle' requires the chroman ring to be reduced back to the non-radical form by the action of the glutathione system. By contrast, the bis-pyrrolo pyrimidine component of U-83836E can repeatedly (catalytically) scavenge lipid peroxyl radicals, the detailed mechanism of which has been reviewed previously (25). The dual mechanism by which U-83836E scavenges lipid peroxyl radicals makes it one of the most potent anti-oxidizing agents available.

The generation of TBARS is a well-established index of lipid peroxidation (26). The formation of lipid peroxyl radicals is considered one of the 'dogmas' of how lipid peroxidation is propagated (27). Lipid peroxidation is reported to be induced by peroxynitrite through its decomposition into hydroxyl radicals (28). Therefore, it is not surprising that the scavenging of lipid peroxyl radicals via the use of U-83836E inhibited the TBARS formation induced by peroxynitrite treatment. The results of the present study demonstrated that the inhibition of TBARS production by U-83836E was more pronounced in the plasma compared with that in the platelet samples. There are several reasons that may explain this finding: i) Platelet samples may have a higher lipid content than plasma samples and thus may produce more lipid peroxidation products upon their treatment with peroxynitrite compared to plasma samples; ii) the assay used to measure TBARS formation only estimates the level of free MDA, therefore, protein-bound MDA does not react with TBA using the assay described (21). It is possible that a scenario exists in which there is a higher free MDA to protein-bound MDA ratio in the platelet samples compared with that in the plasma samples upon their combined treatment with the same dose of peroxynitrite and U-83836E. As the TBARS assay is only able to detect the free MDA, the results suggest a weaker inhibition of TBARS production by $\mathrm{U}-83836 \mathrm{E}$ in the platelet samples; iii) inadvertent overheating of the platelet samples compared with the plasma may produce secondary MDA from the breakdown of hydroperoxides (21); this pool of MDA may evade the antagonizing effects of $\mathrm{U}-83836 \mathrm{E}$ in the platelet samples causing the apparent superior inhibition of TBARS formation by U-83836E in the plasma samples. 
By contrast, protein carbonylation is a well-established index of protein oxidation. An increase in the rate of protein carbonylation by peroxynitrite can result from i) oxidation of the side chains of threonine, lysine and proline into carbonyl groups following their reaction with the reactive oxygen or nitrogen species that result from peroxynitrite treatment, including lipid peroxyl radicals (a ROS) (29); or ii) the reaction of the side chains of histidine, cysteine, and lysine with reactive aldehyde species, for example, 4-hydroxynonenal (4-HNE) and MDA, which result from lipid peroxidation (29). Given the above discussion, it is not surprising that scavenging lipid peroxyl radicals by $\mathrm{U}-83836 \mathrm{E}$ reversed the protein carbonylation of blood plasma and platelets induced by peroxynitrite, as lipid peroxyl radicals are ROS by chemical definition (17) and can increase protein carbonylation directly, as explained above, and/or indirectly, as the formation of lipid peroxyl radicals is an intermediate of the biochemical pathway through which lipid peroxidation occurs, which ends in the formation of 4-HNE and MDA.

In conclusion, the present study provided evidence that scavenging lipid peroxyl radicals via the use U-83836E may be a viable alternative to attenuating the cytotoxic effects of peroxnitrite on blood plasma and on platelet lipids and proteins.

\section{Acknowledgements}

Not applicable.

\section{Funding}

Funding for this the present study was provided through a Sabbatical Leave Research Grant awarded by the Deanship of Research at Jordan University of Science and Technology (grant no. 154/2017).

\section{Availability of data and materials}

The datasets generated and/or analyzed during the present study are available from the corresponding author on reasonable request.

\section{Authors' contributions}

All the authors were involved in the design, analysis of the data, and final review of the manuscript. AGM and OA conceived the study; AGM, MAA, OA and ANA assisted in data collection; MAA performed the statistical analysis; AGM, OA and ANA performed all biochemical measurements; MAA and AGM drafted the manuscript. All authors read and approved the final manuscript.

\section{Ethical approval and consent to participate}

All procedures performed in experiments involving human participants were in accordance with the ethical standards of Jordan University of Science and Technology and King Abdullah University Hospital IRB, and with the 1964 Helsinki declaration and its later amendments or comparable ethical standards. Informed consent was obtained from all individual participants included in the study.

\section{Patient consent for publication}

Not applicable.

\section{Competing interests}

The authors declare that they have no competing interests.

\section{References}

1. Turrens JF: Mitochondrial formation of reactive oxygen species. J Physiol 552: 335-344, 2003.

2. Hakim J: Reactive oxygen species and inflammation. C R Seances Soc Biol Fil 187: 286-295, 1993 (In French).

3. Bergamini CM, Gambetti S, Dondi A and Cervellati C: Oxygen, reactive oxygen species and tissue damage. Curr Pharm Des 10: 1611-1626, 2004.

4. Yu BP: Cellular defenses against damage from reactive oxygen species. Physiol Rev 74: 139-162, 1994.

5. Foyer $\mathrm{CH}$ and Noctor G: Ascorbate and glutathione: The heart of the redox hub. Plant Physiol 155: 2-18, 2011.

6. Pigeolet E, Corbisier P, Houbion A, Lambert D, Michiels C, Raes M, Zachary MD and Remacle J: Glutathione peroxidase, superoxide dismutase, and catalase inactivation by peroxides and oxygen derived free radicals. Mech Ageing Dev 51: 283-297, 1990.

7. Finkel $\mathrm{T}$ and Holbrook NJ: Oxidants, oxidative stress and the biology of ageing. Nature 408: 239-247, 2000.

8. Waris G and Ahsan H: Reactive oxygen species: Role in the development of cancer and various chronic conditions. J Carcinog 5: 14-14, 2006.

9. Benzi G and Moretti A: Are reactive oxygen species involved in Alzheimer's disease? Neurobiol Aging 16: 661-674, 1995.

10. Werner $C$ and Engelhard K: Pathophysiology of traumatic brain injury. Br J Anaesth 99: 4-9, 2007.

11. Hall ED, Vaishnav RA and Mustafa AG: Antioxidant therapies for traumatic brain injury. Neurotherapeutics 7: 51-61, 2010.

12. Houstis N, Rosen ED and Lander ES: Reactive oxygen species have a causal role in multiple forms of insulin resistance. Nature 440: 944-948, 2006

13. Maurya PK, Noto C, Rizzo LB, Rios AC, Nunes SO, Barbosa DS, Sethi S, Zeni M, Mansur RB, Maes M, et al: The role of oxidative and nitrosative stress in accelerated aging and major depressive disorder. Prog Neuropsychopharmacol Biol Psychiatry 65: 134-144, 2016.

14. Gutteridge JM and Halliwell B: The measurement and mechanism of lipid peroxidation in biological systems. Trends Biochem Sci 15: 129-135, 1990.

15. Hemnani T and Parihar MS: Reactive oxygen species and oxidative DNA damage. Indian J Physiol Pharmacol 42: 440-452, 1998.

16. Bandyopadhyay U, Das D and Banerjee RK: Reactive oxygen species: Oxidative damage and pathogenesis. Curr Sci 77: 658-666, 1999.

17. Halliwell B: Reactive oxygen species in living systems: Source, biochemistry, and role in human disease. Am J Med 91 (3C): $14 \mathrm{~S}-22 \mathrm{~S}, 1991$

18. Beckman JS, Beckman TW, Chen J, Marshall PA and Freeman BA: Apparent hydroxyl radical production by peroxynitrite: Implications for endothelial injury from nitric oxide and superoxide. Proc Natl Acad Sci USA 87: 1620-1624, 1990.

19. Pacher P, Beckman JS and Liaudet L: Nitric oxide and peroxynitrite in health and disease. Physiol Rev 87: 315-424, 2007.

20. Mustafa AG, Bani-Ahmad MA, Jaradat AQ and Allouh MZ: Tempol protects blood proteins and lipids against peroxynitrite-mediated oxidative damage. Exp Biol Med (Maywood) 240: 109-112, 2015.

21. Mustafa AG, Al-Shboul O, Alfaqih MA, Al-Qudah MA and Al-Dwairi AN: Phenelzine reduces the oxidative damage induced by peroxynitrite in plasma lipids and proteins. Arch Physiol Biochem: Dec 19, 2017 (Epub ahead of print).

22. Campo GM, Squadrito F, Campo S, Altavilla D, Avenoso A, Ferlito M, Squadrito G and Caputi AP: Antioxidant activity of U-83836E, a second generation lazaroid, during myocardial ischemia/reperfusion injury. Free Radic Res 27: 577-590, 1997. 
23. Willcox JK, Ash SL and Catignani GL: Antioxidants and prevention of chronic disease. Crit Rev Food Sci Nutr 44: 275-295, 2004

24. Hall ED, Detloff MR, Johnson $\mathrm{K}$ and Kupina NC: Peroxynitrite-mediated protein nitration and lipid peroxidation in a mouse model of traumatic brain injury. J Neurotrauma 21: 9-20, 2004

25. Mustafa AG, Singh IN, Wang J, Carrico KM and Hall ED: Mitochondrial protection after traumatic brain injury by scavenging lipid peroxyl radicals. J Neurochem 114: 271-280, 2010.

26. Esterbauer $\mathrm{H}$ and Cheeseman KH: Determination of aldehydic lipid peroxidation products: malonaldehyde and 4-hydroxynonenal. In: Methods in enzymology. Vol 186. Elsevier, New York, NY, pp407-421, 1990.
27. Buettner GR: The pecking order of free radicals and antioxidants: Lipid peroxidation, $\alpha$-tocopherol, and ascorbate. Arch Biochem Biophys 300: 535-543, 1993.

28. Radi R, Beckman JS, Bush KM and Freeman BA: Peroxynitrite-induced membrane lipid peroxidation: The cytotoxic potential of superoxide and nitric oxide. Arch Biochem Biophys 288: 481-487, 1991.

29. Suzuki YJ, Carini M and Butterfield DA: Protein carbonylation. Antioxid Redox Signal 12: 323-325, 2010. 